\title{
Miners' Nystagmus Following Visual Deprivation: A Case Report
}

\section{Background}

Miners' nystagmus was first recognized in the 1870s in coal miners, in whom it developed after they spent years working underground (1). The incidence of nystagmus was estimated at 5\% (2), and its addition to the schedule of industrial diseases in the Workmen's Compensation Act of 1906 embedded it into the legal—but not the medical—literature.

The underlying cause of miners' nystagmus has remained contentious, with theoretical considerations suggesting a foveal inability to adapt to dark vision leading to nystagmus after years of working underground (3) —a similar mechanism to the irregular eye oscillation seen in adult patients with acquired vision loss. However, several additional symptoms in miners, including headaches, breathlessness, hand tremors, and violent body oscillations (4), led to the belief that "occupational neurosis" was a key determinant of miners' nystagmus.

\section{Objective}

To report a patient who developed oscillopsia due to an acquired pendular nystagmus secondary to almost complete and prolonged light deprivation as an extreme behavior to manage migraine-related photophobia.

\section{Case Report}

A 57-year-old right-handed woman presented with throbbing headaches that initially occurred every 3 to 5 weeks and lasted 5 to 6 hours. These gradually increased in severity and frequency and eventually became daily and persistent. The headaches were mainly bilateral, affecting the forehead, nasal bridge, and temple. She had associated motion sensitivity and severe phonophobia and photophobia, requiring her to wear a mask over her eyes to prevent exposure to light when outdoors. She was diagnosed with chronic migraine in 2011 and treated unsuccessfully with more than 8 migraine prophylactics over a 6-year period, but her headaches were eventually controlled with botulinum toxin and intravenous magnesium sulfate.

In 2017, she reported gradual-onset oscillopsia (“jumping” vision) associated with a halo around objects. She acknowledged that she had been living in continuous darkness for almost 2 years; her 
home was completely blacked out, with only a small camping light set to nocturnal settings for illumination.

Videonystagmography in the dark revealed binocular pendular torsional nystagmus in the primary position with occasional rapid upbeat jerk nystagmus (Supplement Video, available at Annals.org). Eye movements appeared conjugate, and the pendular nystagmus increased in amplitude with changes in gaze. The remainder of the oculomotor assessment (in the dark) was normal. Ophthalmologic assessment at the time of the nystagmus identified normal acuities (6/5-3 right, 6/6-2), color vision, and visual fields. Her fundi were normal, but nystagmus was observed on slit lamp on very dim light. A structural magnetic resonance imaging (MRI) scan of the brain was normal.

We made a provisional diagnosis of acquired pendular nystagmus secondary to visual pathway deafferentation and placed the patient on a program of gradually increasing light exposure using dimmer switches and a lux monitor. By week 5, she was able to tolerate 1 lux for 8 hours a day, and by week 13, she could tolerate 50 lux for 12 consecutive hours a day. Her nystagmus and oscillopsia resolved completely by 8 months (Supplement Video), highlighting the requirement for sensory feedback for normal visual function and the plastic nature of sensory pathways when sensory feedback is removed or reintroduced.

\section{Discussion}

We propose that this patient's nystagmus was caused by temporary deafferentation of cone cells secondary to the self-induced light deprivation. Perfusion imaging in our patient hinted at abnormal blood flow in the occipital cortex (Figure), which was likely a consequence of the visual deprivation and due in turn to pathology of anterior visual pathways. Blood flow changes followed the clinical recovery over time (Figure, bottom), adding further weight to this hypothesis. Electroretinography and dark adaptometry were not performed in this patient, so we cannot comment on rod cell function, although peripheral vision was normal.

This unique case is reminiscent of coal miners' nystagmus and provides evidence of a neurobiological cause. Although British miners usually spent years in dim work environments before symptom onset, they did not experience as much light deprivation as this patient. Many considered miners' nystagmus to be "malingering" with self-induction of "voluntary nystagmus," but in this patient, the absence of 
convergent effort and multiplanar persistent eye oscillations that were pendular rather than saccadic argue against this interpretation (5).

This case highlights how the damaging physiologic effects of visual deprivation can be reversed through inherent neuroplasticity, encompassing elements from social science, psychology, biology, and neuroscience that lie at the core of human behavior.

Salwa Kamourieh, $\mathrm{PhD}$

National Hospital for Neurology and Neurosurgery; London, United Kingdom

Magdalena Sokolska, $\mathrm{PhD}$

UCL Queen Square Institute of Neurology; London, United Kingdom

0000-0003-1715-5263

Harry Akram, MSc

Royal National Ear Nose and Throat \& Eastman Dental Hospital; London, United Kingdom Jay Patel, MSc

Royal National Ear Nose and Throat \& Eastman Dental Hospital; London, United Kingdom Hans Rolf Jäger, PhD

UCL Queen Square Institute of Neurology; London, United Kingdom

0000-0002-6403-4184

Qadeer Arshad, PhD

University of Leicester, United Kingdom

Manjit Matharu, $\mathrm{PhD}$

National Hospital for Neurology and Neurosurgery; London, United Kingdom 0000-0002-4960-2294

Diego Kaski, $\mathrm{PhD}$

National Hospital for Neurology and Neurosurgery, Royal National Ear Nose and Throat \& Eastman Dental Hospital, and UCL Institute of Neurology; London, United Kingdom 0000-0002-0591-646X 
Acknowledgment: The authors thank the patient and her partner for allowing them to publish her story; the neuro-ophthalmology department at the National Hospital for Neurology and Neurosurgery, London, for performing visual assessments; and Professor David Zee for providing invaluable historical data.

Financial Support: Professor Kaski is supported by a grant from the Meniere's Society and by the National Institute for Health Research University College London Hospitals Biomedical Research Centre.

Disclosures: Disclosures can be viewed at www.acponline.org/authors/icmje/ConflictOfInterestForms.do?msNum=L20-1261.

Corresponding Author: Diego Kaski, MBBS, PhD, Department of Clinical and Motor Neurosciences, University College London, 5th Floor, 33 Queen Square, London WC1N 3BG, United Kingdom; e-mail, d.kaski@ucl.ac.uk.

\section{References}

1. Renton JC. On a case of miner's nystagmus. Glasgow Med J. 1879;11:202-3. [PMID: 30433118]

2. Ohm J. [The frequency as the most important characteristic of miner's nystagmus. II. The relations of the frequency to the age and duration of mining]. Albrecht Von Graefes Arch Ophthalmol. 1952;153:321-32. [PMID: 13040236]

3. Fishman RS. Dark as a dungeon: the rise and fall of coal miners' nystagmus. Arch Ophthalmol. 2006;124:1637-44. [PMID: 17102014]

4. O’Sullivan F. Miner's Nystagmus: Its Symptoms, Etiology, and Treatment (With Notes on Fifty Cases). John Wright \& Sons; 1933.

5. Kaski D, Bronstein AM, Edwards MJ, et al. Cranial functional (psychogenic) movement disorders. Lancet Neurol. 2015;14:1196-205. [PMID: 26581970] doi:10.1016/S1474-4422(15)00226-4

Figure. Perfusion imaging at baseline $(\mathrm{T}=0)$ and 8 months $(\mathrm{T}=8 \mathrm{~m})$. Left. Perfusion-weighted images derived from arterial spin labeling 3-Tesla magnetic resonance imaging at the first clinical assessment (top) and 8 months later (bottom). The initial study shows serpiginous hyperintense areas 
overlying the occipital lobes (left more than right [red circle]), which corresponds to labeled blood in leptomeningeal arteries that has not yet fully reached the brain parenchyma. At 8 months, this is much less prominent, and the signal over the visual cortices is more homogeneous compared with the baseline scan. Right. Arterial transit time maps. Colors represent increasing arterial transit time in seconds, from blue to red. At baseline (top), there is marked prolongation of arterial transit times over the occipital lobes and visual cortical areas compared with the remainder of the brain, particularly on the left (red circle), coinciding with the presence of maximal nystagmus. At 8 months (bottom), the arterial transit time is considerably less delayed (shift from red to green [red circle]), likely secondary to increased visual cortical perfusion compared with baseline.

Supplement Video. Videonystagmography performed with the patient looking straight ahead (center gaze). The recordings were taken in the light (with fixation) and the dark (without fixation) at each time point. Removal of fixation increases the amplitude and intensity of nystagmus, making this easier to visualize. The first half of this recording was done with fixation, and the second half was done without fixation. Note that the patient had not started graded light reexposure therapy in the top video; had been exposed to partial light in the middle video; and was tolerating low-level daylight in the bottom video, where there is no nystagmus. 